\title{
Tumor necrosis factor receptor-associated factor 6 (TRAF6) inhibition modulates bone loss and matrix metalloproteinase expression levels in collagen-induced rheumatoid arthritis rat
}

\author{
Jiangtao Guo ${ }^{1 \#}$, Xuqing Cao ${ }^{2 \#}$, Xiaoli Ma ${ }^{1}$, Chunfang Hao ${ }^{1}$, Lili Wu ${ }^{1}$, Mingzhu Zhang ${ }^{1}$, Yashan Yang ${ }^{1}$, \\ Jingtian Zhao ${ }^{1}$, Kunting Chen ${ }^{1}$, Zhe Yin ${ }^{1}$ \\ ${ }^{1}$ Department of Rheumatology and Immunology, People's Hospital of Ningxia Hui Autonomous Region (the Affiliated People's Hospital of Ningxia \\ Medical University and the First Affiliated Hospital of Northwest Minzu University), Ningxia, China; ${ }^{2}$ Department of Neurology, People's Hospital \\ of Ningxia Hui Autonomous Region (the Affiliated People's Hospital of Ningxia Medical University and the First Affiliated Hospital of Northwest \\ Minzu University), Ningxia, China \\ Contributions: (I) Conception and design: J Guo, X Cao; (II) Administrative support: X Ma, C Hao, L Wu; (III) Provision of study materials or patients: \\ J Guo, X Cao, X Ma, C Hao, J Zhao, K Chen, Z Yin; (IV) Collection and assembly of data: All authors; (V) Data analysis and interpretation: J Guo, X \\ Cao, L Wu, M Zhang, Y Yang, J Zhao, K Chen, Z Yin; (VI) Manuscript writing: All authors; (VII) Final approval of manuscript: All authors. \\ \#These authors contributed equally to this work as co-authors. \\ Correspondence to: Xuqing Cao. Department of Neurology, People's Hospital of Ningxia Hui Autonomous Region (the Affiliated People's Hospital of \\ Ningxia Medical University and the First Affiliated Hospital of Northwest Minzu University), Zhengyuan North Street Ziyun Hua Ting 14-4-302, \\ Jinfeng District, Ningxia, China. Email: 13909592466@163.com.
}

Background: Rheumatoid arthritis (RA) is a main characterized by persistent synovitis, systemic inflammation, and autoantibodies. Tumor necrosis factor receptor-associated factor 6 (TRAF6) is an E3 ubiquitin ligase and is a crucial cytoplasm signal adaptor that can regulate critical biological processes. This research aims to explore the function of TRAF6 on bone loss and matrix metalloproteinase (MMP) expression in collagen-induced RA rats.

Methods: The RA model in rats (Sprague Dawley rat, 5-6 weeks old, weight $246.88 \pm 8.31 \mathrm{~g}$ ) was set up via using collagen-induced RA. The shRNA-TRAF6 knockdown efficiency was tested using real-time reverse transcription-polymerase chain reaction (qRT-PCR) and western blot, respectively. The rats were divided into four groups: the control group, RA group, RA + shRNA-NC group, and RA + TRAF6-shRNA group. The tartrate-resistant acidic phosphatase (TRAP), hematoxylin and eosin (H\&E), and Saffron O staining were employed to test the bone injury. The mRNA and protein expressive of Osteoclast-associated receptor (OSCAR), TRAP, Osterix (OSX), Collagen type I alpha 1 (COL1A1), Distal-less homeobox2 (Dlx2), tissue inhibitor of metalloproteinase (TIMP), matrix metalloproteinase-1(MMP-1), Cyclooxygenase 2 (COX2) and qRT-PCR performed MMP-13 and western blot, respectively.

Results: The mRNA and protein expression levels of TRAF6 were down-regulated in the RA + TRAF6shRNA group. After the levels of TRAF6 were inhibited, the levels of bone volume/total volume (BV/TV), trabecular bone thickness (Tb.Th), and trabecular bone number (Tb.N) were increased, while the levels of trabecular bone space (Tb.Sp), Osteocalcin and ALP were deceased. The mRNA and protein expression levels of OSCAR, TRAP, MMP-1, COX2, and MMP-13 were reduced obviously in the RA + TRAF6shRNA group compared with the RA + shRNA-NC group, while the levels of TIMP-1, OSX, CoL1A1, and DLx2 were enhanced obviously.

Conclusions: Inhibition of TRAF6 reduces bone loss and MMP expression levels in collagen-induced RA rat, and supplies an alternative treatment method in RA.

Keywords: tumor necrosis factor receptor-associated factor 6 (TRAF6); rheumatoid arthritis (RA); bone loss; matrix metalloproteinase expression 
Submitted Sep 03, 2020. Accepted for publication Oct 28, 2020.

doi: 10.21037/apm-20-1894

View this article at: http://dx.doi.org/10.21037/apm-20-1894

\section{Introduction}

Rheumatoid arthritis (RA) is a main characterized by persistent synovitis, systemic inflammation, and autoantibodies. Women, smokers, and those with a family history of the disease are most often affected (1). Methotrexate is the drug of choice to treat RA, sometimes combined with biological agents, including tumor necrosis factor (TNF) inhibitors (2). However, even with these treatment options, including disease-altering anti-rheumatic drugs and available biological agents, many patients still cannot adequately control their disease (3). Radiation synovial replacement, synovectomy, or axial correction with tendon transfer are essential methods for surgical treatment of RA in earlier stages (4). Many recent advancements in pharmacologic therapy have improved RA outcomes significantly, and many further patients can be treated. However, the improvement of RA results is accompanied by substantial financial costs (5). In traditional RA treatment, due to the short biological half-life and poor bioavailability, high doses and frequent doses is require. These anti-RA medications may cause serious side effects on extra-articular tissues. Studies have shown that nanotechnology has become a promising tool for the development of new drug delivery systems for the treatment and diagnosis of intractable diseases such as rheumatoid arthritis $(6,7)$. At present, RNA interference (RNAi) is a powerful endogenous process initiated by short double-stranded RNAs, which results in sequence-specific posttranscriptional gene silencing. The possibility of blocking the expression of any protein carries huge expectations for potential therapeutic applications in a wide range of diseases (8). Therefore, improving the treatment of RA is an integral part of our future research.

Tumor necrosis factor receptor-associated factor 6 (TRAF6) is a significant binding protein in the TRAF family and can regulate the TNF receptor superfamily. The interleukin 1 receptor/Toll-like receptor family, and is considered a vital participant in regulating inflammation and immunity (9-11). TRAF6 is necessary for many biological processes, including the chronic innate immune signaling with myelodysplasia (12), anti-myeloma and antibone resorptive (13), tumor angiogenesis (14), and cancer metastasis $(15,16)$. Notably, the overexpression of TRAF6 in the synovium is related to the increase of compensatory bone formation. Therefore, TRAF6 is engaged in the pathogenesis of bone metabolic imbalance via regulating the synovial inflammation of RA (17). Sinomenine suppresses expression levels of differentiation factor 88 (MyD88) and TRAF-6 in RA-Fibroblast-like synoviocytes (FLSs) (18). Tomatidine inhibits osteoclast production and reduces bone loss caused by estrogen deficiency by regulating TRAF6-mediated signal transduction (19). However, downregulation of TRAF- 6 plays the role is not reported in osteoclast, osteoblast, and chondrocyte.

We present the following article in accordance with the ARRIVE reporting checklist (available at http://dx.doi. org/10.21037/apm-20-1894).

\section{Methods}

\section{Animals}

Sixty Sprague Dawley (SD) rats (5-6 weeks old, weight $246.88 \pm 8.31 \mathrm{~g})$ were retrieved from the Shanghai Laboratory Animal Center, Chinese Academy of Sciences (Shanghai, China). All animal care and handling were performed considering the National Institutes of Health (NIH) Guidelines for the Care and Use of Laboratory Animals and were approved by the People's Hospital of Ningxia Hui Autonomous Region. The research was reviewed and approved by the Ethics Committee for Experimental Animal Management and Animal Welfare of People's Hospital of Ningxia Hui Autonomous Region. Rats were housed individually in stainless steel cages with glass water bottles. Rats were held for adaptation for $14 \mathrm{~d}$ before collagen-induced RA. Rats were kept in a room under the following conditions: temperature of $23 \pm 1{ }^{\circ} \mathrm{C}$, the humidity level of 50-60\%, and the 12 hours light/dark cycle.

\section{$R A$ model rats}

Of the sixty rats, 15 rats were randomly selected as the control group, and the remaining 45 rats were selected as the RA group. The RA model in rats was set up via using collagen-induced RA. So rats were divided randomly into four groups: control group, RA group, RA + shRNA- 
NC group and RA + shRNA-TRAF6 group. Type II bovine collagen was dissolved in $0.05 \mathrm{~mol} / \mathrm{L}$ acetic acid at a concentration of $2 \mathrm{mg} / \mathrm{mL}$ and then emulsified in an equal volume of complete Freund's adjuvant (CFA; Jon Drex, Washington, USA). Rats were immunized intracutaneously with $200 \mu \mathrm{L}$ emulsion divided into four points symmetrically along the back. After seven days, a similar amount of bovine collagen type II emulsified into CFA injected intracutaneously as a booster dose (20). The severity of collagen-induced RA was assessed using a scoring system (21). Through this method, we judge whether the RA was set up successfully.

\section{shRNA-mediated TRAF6 knockdown}

Following manufacturer's instructions, peripheral blood mononuclear cells (PBMC) were retrieved from the peripheral blood in the RA patients and healthy controls. In short, Ficoll immediately isolated PBMC (TBD Science, Tianjin, China), and then resuspended at a cell concentration of $10^{5} / \mathrm{mL}$ in RPMI- 1640 medium containing $10 \%$ fetal bovine serum (FBS). HANBIO Company designed the effective TRAF6-shRNA sequence. The HANBIO Company synthesized the TRAF6shRNA lentiviral vector (Shanghai, China). An shRNA$\mathrm{NC}$ was used as a control for all experiments. After 24 hours, TRAF6-shRNA and shRNA-NC lentiviral vector were transfected into $\mathrm{PBMC}$ with lipofectamine 3000 reagents (Life Technologies Corporation) considering the manufacturer's instructions. The shRNA knockdown efficiency was evaluated using Real-time reverse transcription-polymerase chain reaction (qRT-PCR) and western blot.

\section{qRT-PCR}

According to manufacturers' protocol, total RNA was isolated by employing the TRIzol reagent kit (Invitrogen, Beijing, China). The total RNA concentration was measured by adopting the Gene Quant ProRNA/DNA Calcula-tor (Amersham Pharmacia Biotec, UK). The PrimeScript RT reagent Kit (TakaRa, Dalian, China) was used to perform reverse transcription. The 2 SYBR Premix Ex Taq ${ }^{\mathrm{TM}}$ II (TakaRa, Dalian, China) was employed to assemble the reaction system of qRT-PCR. The reaction system is carried out in the Bio-Rad CFX-96 (Bio-Rad, CA, USA). GAPDH was used for normalizing. The qRT-PCR data were analyzed using the $2^{-\Delta \Delta \mathrm{Ct}}$ method to calculate the relative expression levels of mRNA. Primer sequences were used in this study in Table 1.

\section{Western blot assay}

The RIPA lysis buffer (Beyotime Institute of Biotechnology, Shanghai, China) was employed to extract proteins in the bone homogenate following the manufacturer's protocol. The BCA Protein Assay Kit were retrieved from Biyuntian Biological Technology Co., Ltd. (Shanghai, China) and were employed to measure the protein concentrations. Primary antibody (Table 2) were integrated with the targeted protein with incubation at room temperature for 1 hour. Secondary antibodies conjugated with goat anti-rabbit IgG horseradish peroxidase (HRP)-conjugated secondary antibodies for onehour. The band densities were decided and analyzed with an automatic digital gel image analysis system Bio-Rad CFX-96 (Bio-Rad, CA, USA).

\section{Bone morphometry}

Rat femurs were dissected free of soft tissue, fixed in $4 \%$ paraformaldehyde for 24 hours, and scanned under micro-computed tomography $(\mu \mathrm{CT})$. The morphometry of trabecular bones was assessed by high-resolution Inveon microtomography (Siemens, Munich, Germany). Parameters included bone volume/total volume (BV/TV), trabecular bone thickness (Tb.Th), trabecular bone number (Tb.N), and trabecular bone space (Tb.Sp).

\section{Histological examination}

Bone samples were fixed with $10 \%$ neutral buffered formalin. Samples were then decalcified in diethylpyrocarbonate treated $0.2 \mathrm{M}$ ethylenediaminetetraacetic acid (EDTA), embedded in paraffin wax, cut into sections (5 $\mu$ m-thick), and stained with tartrate-resistant acidic phosphatase (TRAP), hematoxylin and eosin (H\&E), and Saffron O staining.

\section{Statistical analysis}

Each experiment in this study was conducted three times. The data were presented as mean \pm SD. Statistical analyses between two groups were conducted using Student's $t$-tests and SPSS 25.0 software (IBM, Armonk, USA). The significance of differences between treatment groups was studied using a one-way analysis of variance (ANOVA). A P 
Table 1 Primer sequences

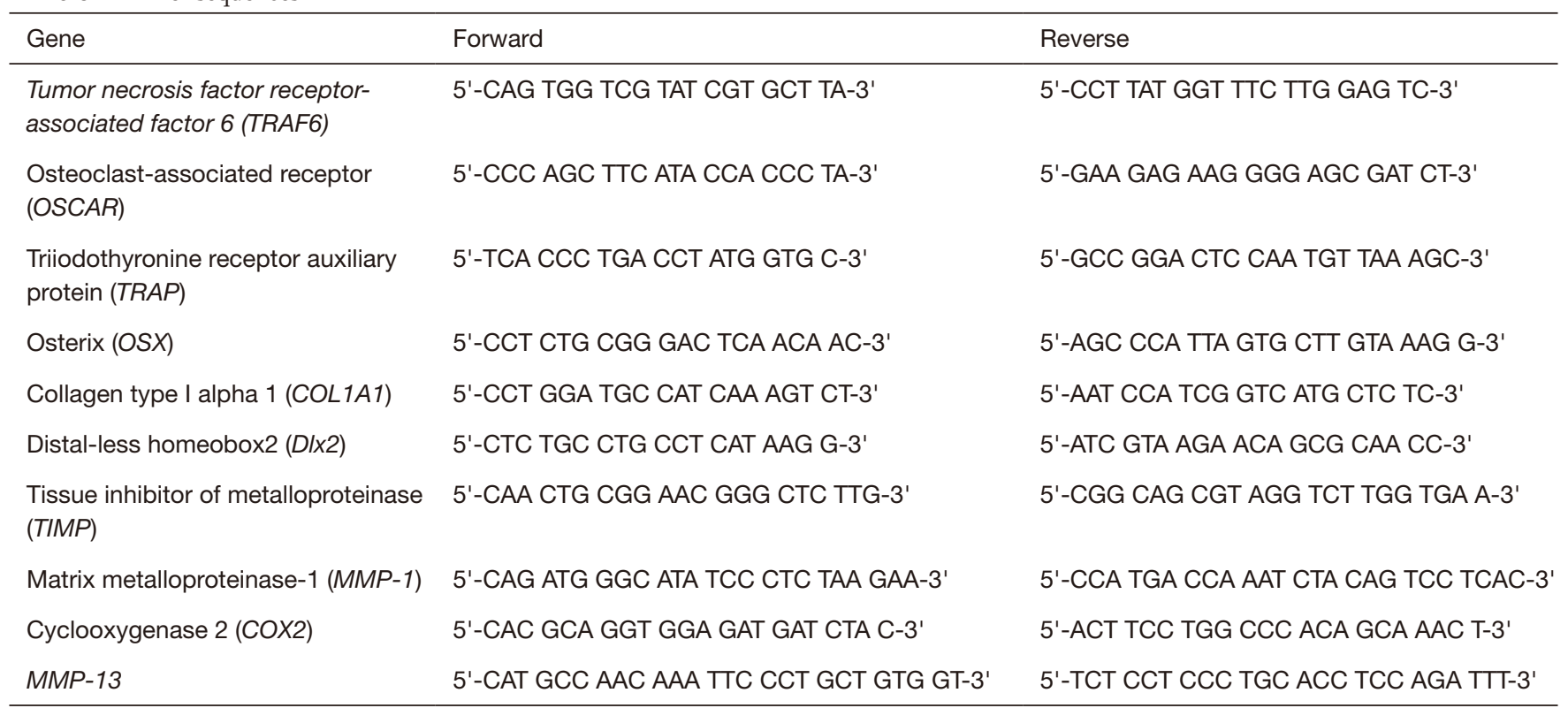

Table 2 Antibody information

\begin{tabular}{ll}
\hline Antibody & Antibody information \\
\hline anti-TRAF6 & $1: 1,000, \# 8028$, Cell Signaling \\
anti-OSCAR & $1: 1,000$, sc-34233, Santa Cruz Biotechnology \\
anti-TRAP & $1: 1,000, \# 15094$, Cell Signaling \\
anti-OSX & $1: 1,000$, ab209484, abcam \\
anti-COL1a1 & $1: 1,000, \# 39952$, Cell Signaling \\
anti-DLX2 & $1: 5,000$, ab272902, abcam \\
anti-TIMP & $1: 1,000, \# 8946$, Cell Signaling \\
anti-MMP-1 & $1: 1,000, \# 54376$, Cell Signaling \\
anti-COX2 & $1: 1,000, \# 12282$, Cell Signaling \\
anti-MMP13 & $1: 1,000, \# 69926$, Cell Signaling \\
\hline
\end{tabular}

value of $<0.05$ was showing statistical significance.

\section{Results}

\section{TRAF6 expression in collagen-induced $R A$ rat}

Considering previous research, TRAF6 plays a direct role in the proinflammatory effects and proliferation of RA FLSs (22). As shown in Figure 1, the mRNA and protein expression levels of TRAF6 were up-regulated, obviously in the RA group compared with the control group $(\mathrm{P}<0.05)$. TRAF6 in RA was inhibited using Lentiviral-TRAF6-
shRNA transfection. The mRNA and protein expression levels of TRAF6 were down-regulated, obviously in the RA + TRAF6-shRNA group compared with the RA + shRNA$\mathrm{NC}$ group $(\mathrm{P}<0.05)$.

\section{Effect of down-regulation of TRAF6 on bone-specific indicators}

As shown in Figure 2, the levels of BV/TV, Tb.Th and Tb.N were deceased in the RA group compared with the control group, while the levels of Tb.Sp, Osteocalcin, and ALP were increased $(\mathrm{P}<0.05)$. However, after the levels of TRAF6 were inhibited, the levels of BV/TV, Tb.Th and Tb.N were increased in the RA + TRAF6-shRNA group compared with the RA + shRNA-NC group $(\mathrm{P}<0.05)$, while the levels of Tb.Sp, Osteocalcin, and ALP were decreased $(\mathrm{P}<0.05)$. In summary, inhibition of TRAF6 might break the balance between osteoclast and osteoblast production.

\section{Effect of down-regulation of TRAF6 on osteoclast production}

As shown in Figure 3A, TRAP staining detected osteoclast production. The result showed that the number of osteoclasts was increased significantly in the RA group compared with the control group. However, after the levels of TRAF6 were inhibited, the number of osteoclasts 

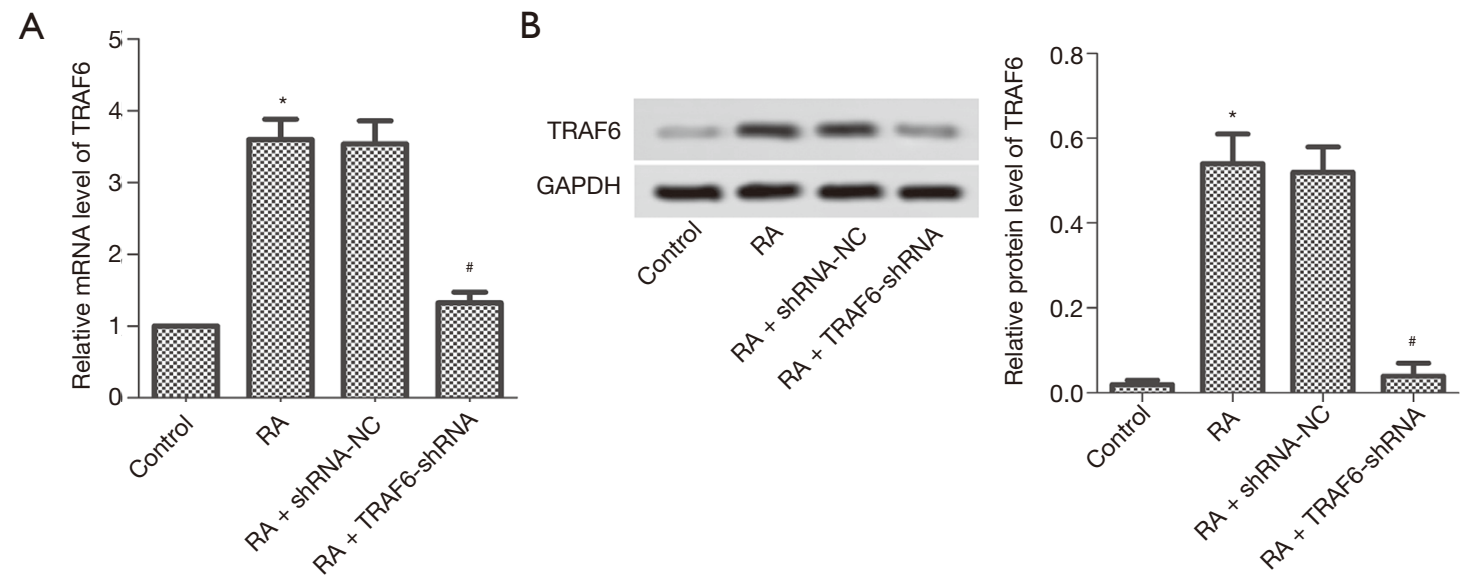

Figure 1 TRAF6 expression in collagen-induced rheumatoid arthritis rat. (A) The mRNA expressive level of TRAF6 was performed by qRT-PCR; (B) the expressive protein levels of TRAF6 were performed by western blot. *, P<0.05 compared with the control group; *, $\mathrm{P}<0.05$ compared with the RA + shRNA-NC group. TRAF6, tumor necrosis factor receptor-associated factor 6; qRT-PCR, real-time reverse transcription-polymerase chain reaction; .

was decreased significantly in the RA + TRAF6-shRNA group compared with the RA + shRNA-NC group. Also, as shown in Figure $3 B, C$, the mRNA and protein expression levels of OSCAR and TRAP were enhanced obviously in the RA group compared with the control group $(\mathrm{P}<0.05)$. However, after the levels of TRAF6 were inhibited, the mRNA and protein expression levels of OSCAR and TRAP were reduced in the RA + TRAF6-shRNA group compared with the RA + shRNA-NC group $(\mathrm{P}<0.05)$. In summary, inhibition of TRAF6 might suppress osteoclast production.

\section{Effect of down-regulation of TRAF6 on osteoblast production}

As shown in Figure 4A, HE stains showed that osteoblast loss was observed in the RA group compared with the control group. However, after the levels of TRAF6 were inhibited, HE stains showed that osteoblast loss was recovered in the RA + TRAF6-shRNA group compared with the RA + shRNA-NC group. The osteoblast production was detected by Saffron $\mathrm{O}$ staining. The result showed that the number of osteoblasts was suppressed significantly in the RA group compared with the control group. However, after the levels of TRAF6 were inhibited, the number of osteoblasts was elevated significantly in the RA + TRAF6-shRNA group compared with the RA + shRNA-NC group. The contents of cartilage (Figure $4 B$ ) and trabecular bone (Figure $4 C$ ) were markedly decreased in the RA group compared with the control group, but were increased after the levels of TRAF6 were inhibited in the RA + TRAF6-shRNA group compared with the RA + shRNA-NC group $(\mathrm{P}<0.05)$. The mRNA and protein expression levels of OSX, CoL1A1, and DLx2 were detected by qRT-PCR and western blot (Figure $4 D, E$ ). The mRNA and protein expression levels of OSX, CoL1A1, and DLx2 were reduced in the RA group compared with the control group $(\mathrm{P}<0.05)$. However, after the levels of TRAF6 were inhibited, The mRNA and protein expression levels of OSX, CoL1A1, and DLx2 were enhanced obviously in the RA + TRAF6-shRNA group compared with the RA + shRNA-NC group $(\mathrm{P}<0.05)$. In brief, inhibition of TRAF6 might promote osteoblast production.

\section{Effect of down-regulation of TRAF6 on MMPs}

As shown in Figure 5, the mRNA and protein expression levels of TIMP-1, MMP-1, COX2, and qRT-PCR detected MMP-13 and western blot (Figure 5A,B). The mRNA and protein expression levels of TIMP-1 were reduced obviously in the RA group compared with the control group while increasing the mRNA and protein expression levels of MMP-1, COX2, and MMP-13 ( $\mathrm{P}<0.05)$. However, after the levels of TRAF6 were inhibited, The mRNA and protein expression levels of TIMP-1were enhanced obviously in the RA + TRAF6-shRNA group compared with the RA + shRNA-NC group, while decreasing mRNA and protein 

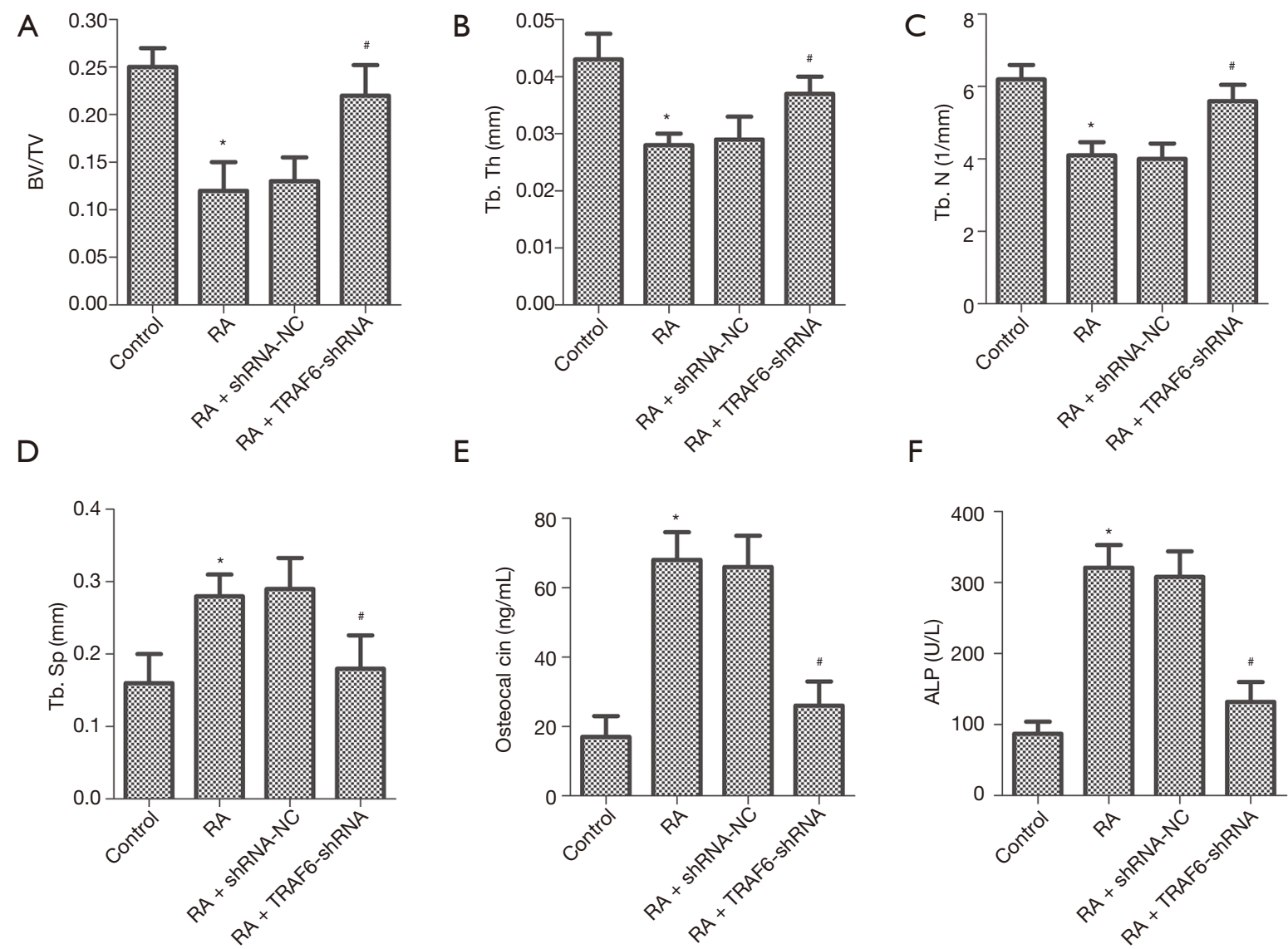

Figure 2 Effect of down-regulation of TRAF6 on bone-specific indicators. The statistical analysis of (A) BV/TV, (B) Tb.Th (mm), (C) Tb.N (mm), (D) Tb.Sp (mm), (E) osteocalcin (ng/mL) and (F) ALP (U/L). *, P<0.05, vs. control group; *, P<0.05, vs. RA + shRNA-NC group. TRAF6, tumor necrosis factor receptor-associated factor 6; BV/TV, bone volume/total volume; Tb.Th, trabecular bone thickness; Tb.N, trabecular bone number; Tb.Sp, trabecular bone space.

expression levels of TIMP-1, MMP-1, COX2, and MMP$13(\mathrm{P}<0.05)$. In summary, inhibition of TRAF6 might improve MMPs production.

\section{Discussion}

There are three significant players directly responsible for the pathogenesis of rheumatoid joint destruction, including the Synovial mesenchymal cells, matrix metalloproteinases (MMPs), and osteoclasts (23). The BV/TV value reflects the distribution density of trabecular bone and is an important index used to evaluate trabecular bone microstructure. In our research, the levels of BV/TV, Tb.Th and Tb.N were decreased in the RA group, while the levels of Tb.Sp, Osteocalcin, and ALP were increased. However, after the levels of TRAF6 were inhibited, the levels of BV/TV, Tb.Th and Tb.N were increased in the RA + TRAF6-shRNA group compared with the RA + shRNA-NC group, while the levels of Tb.Sp, Osteocalcin, and ALP were decreased. These results suggested that inhibition of TRAF6 broke the balance between osteoclast and osteoblast production.

It is widely known that osteoclasts have a unique ability to destroy bone and play a vital function in homeostatic bone remodeling and arthritic bone erosion. Osteoclasts are immune cells, not only mediating the bone destruction but also are involved in immune response (24). Recently, it was demonstrated that Osteoclasts play a critical role in bone destruction in RA. RA synovial tissue provides a suitable microenvironment for the differentiation of monocytemacrophage lineage cells into osteoclasts is the main reason. Simultaneously, the formation of osteoclasts is induced by nuclear factor receptor activator kappa B ligand (RANKL) plays a critical regulatory role in the bone destruction of RA (25). Importantly, TRAF6 ubiquitin ligase is necessary 
A

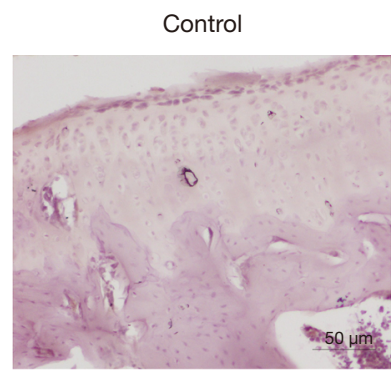

RA

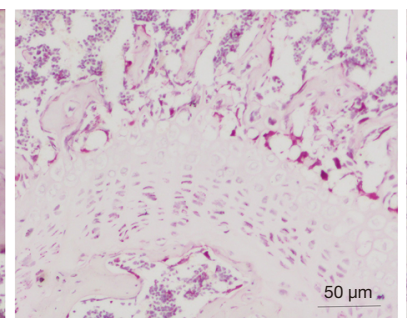

$\mathrm{RA}+$ shRNA-NC

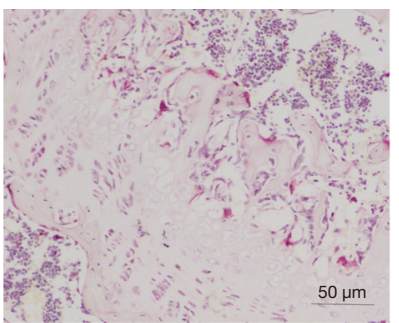

RA + TRAF6-shRNA

B

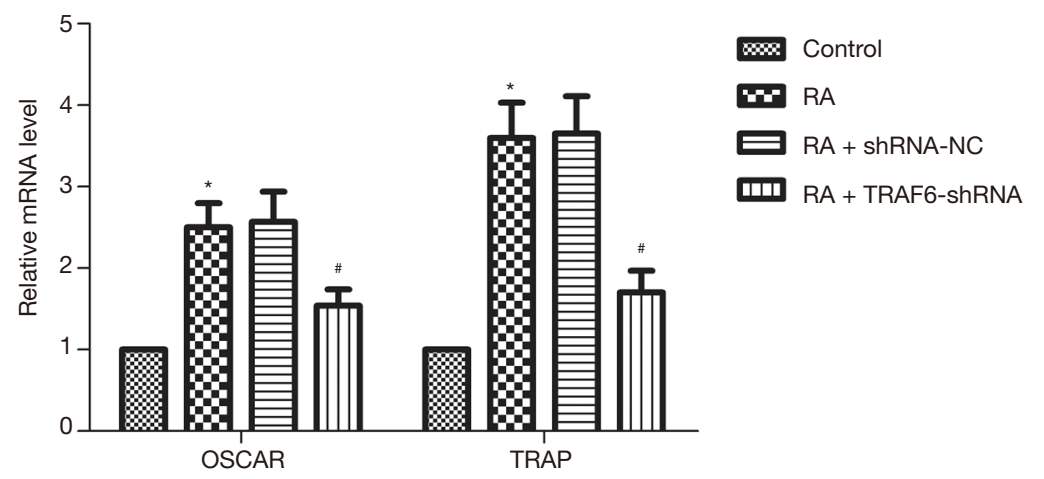

C

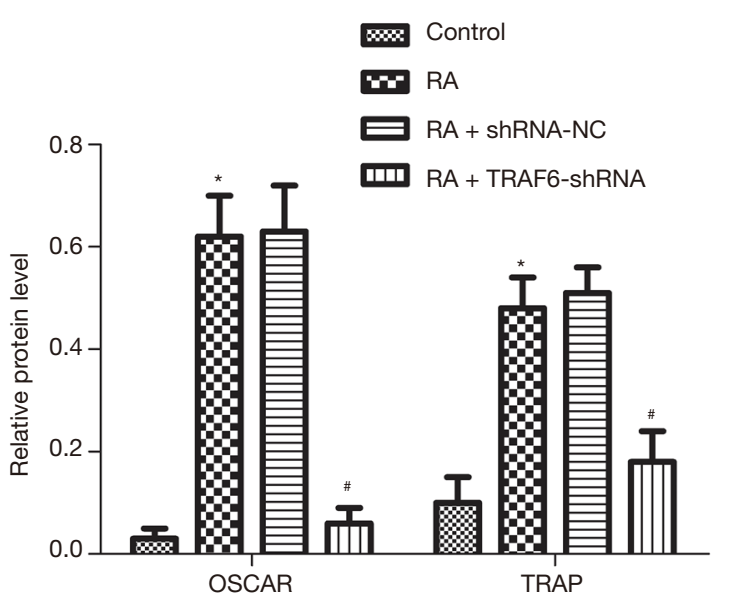

Figure 3 Effect of down-regulation of TRAF6 on osteoclast production. (A) TRAP staining detected the osteoclast production. Magnification 400×; (B) the mRNA expressive levels of OSCAR and TRAP were performed by qRT-PCR; (C) the expressive protein levels of OSCAR and TRAP were performed by western blot. *, $\mathrm{P}<0.05$, vs. control group; " $\mathrm{P}<0.05, v s$. RA + shRNA-NC group. TRAF6, tumor necrosis factor receptor-associated factor 6; TRAP, tartrate-resistant acidic phosphatase; OSCAR, osteoclast-associated receptor; qRT-PCR, real-time reverse transcription-polymerase chain reaction.

for RANKL signaling and differentiation of osteoclast (26). Previous research has demonstrated an association between a genetic variant of TRAF6 and low bone mineral density (BMD) among patients with RA (27).

Elevated synovial expression of TRAF6 is related to the severity of synovitis and density of CD68+ cell, and takes part in the pathogenesis of RA synovitis and differentiation of osteoclast (28). In our research, the down-regulation of TRAF6 significantly decreased the number of osteoclasts in TRAP staining. Also, after the levels of TRAF6 were 
A
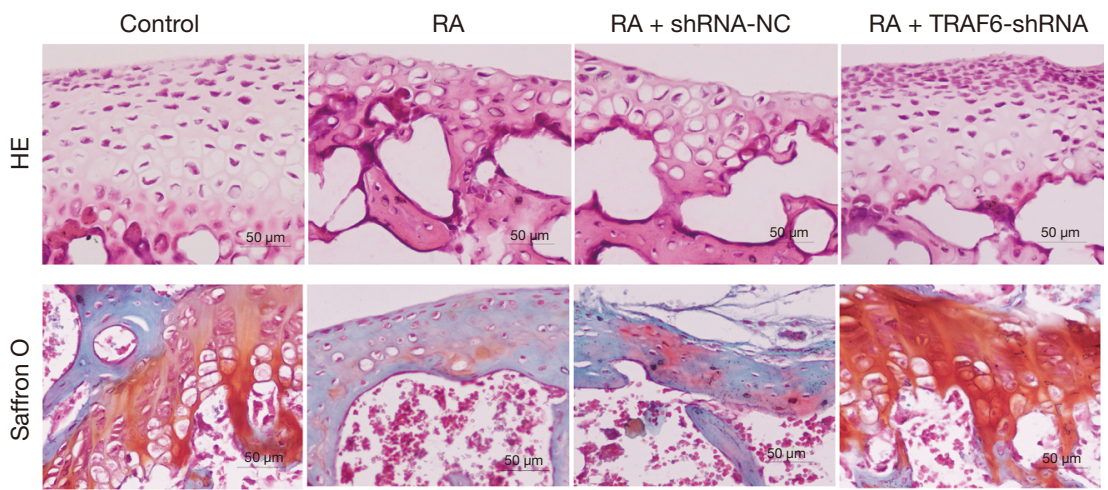

B

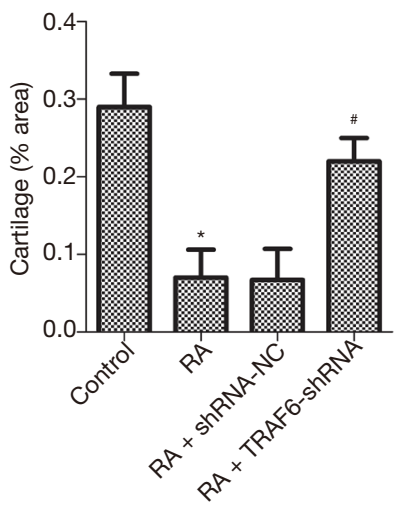

C

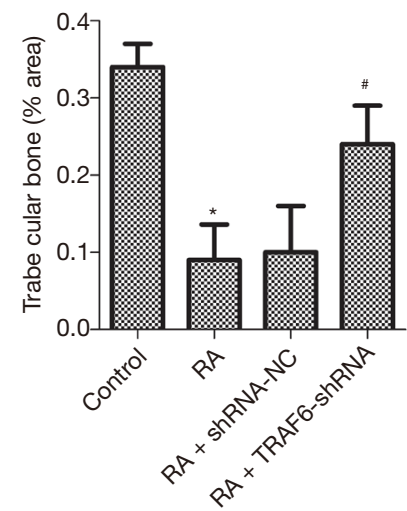

D
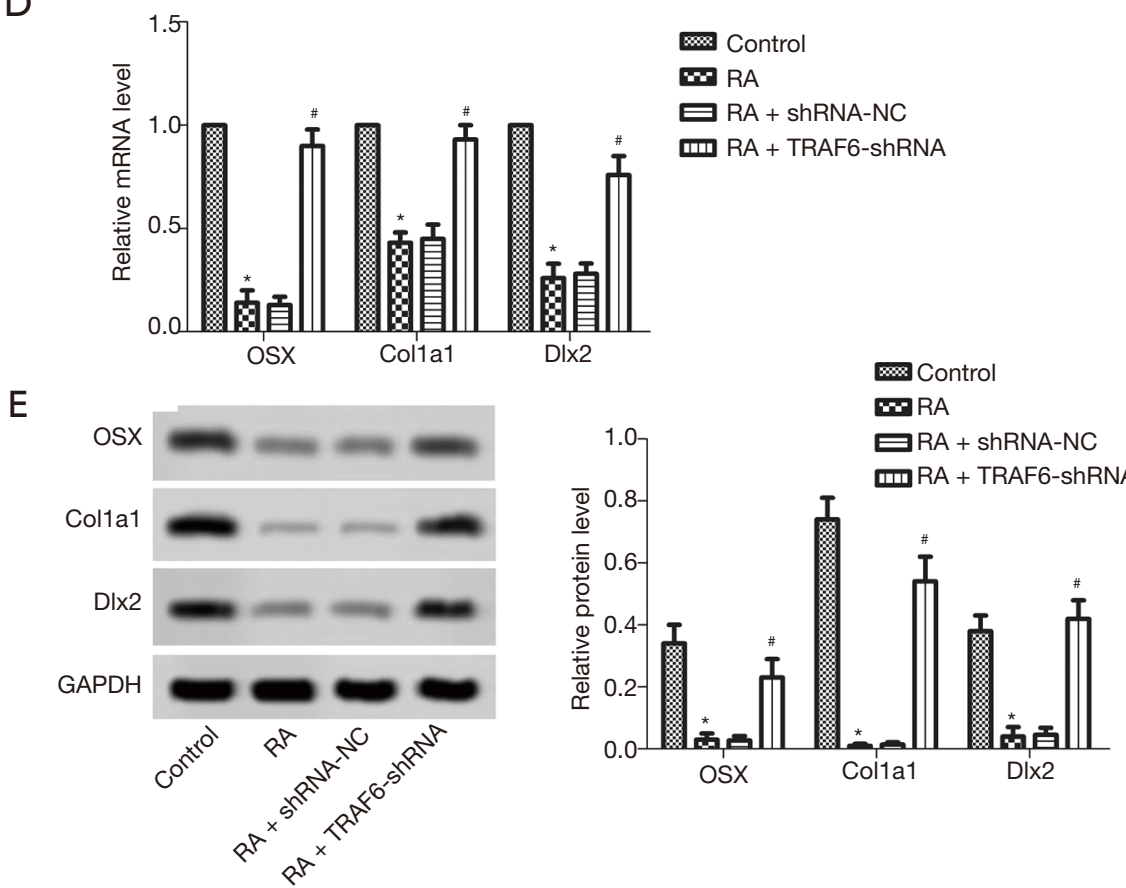

Figure 4 Effect of down-regulation of TRAF6 on osteoblast production. (A) HE stains. The osteoblast production was detected by Saffron O staining. Magnification 400x. The relative area of collagen (B) and trabecular bone (C) were analyzed using Image J software. (D) The mRNA expressive levels of OSX, CoL1A1, and DLx2 were performed by qRT-PCR. (E) The expressive protein levels of OSX, CoL1A1, and DLx2 were performed by western blot. * $\mathrm{P}<0.05$, vs. control group; ${ }^{*}, \mathrm{P}<0.05$, vs. RA + shRNA-NC group. TRAF6, tumor necrosis factor receptor-associated factor; OSX, osterix; COL1A1, Collagen type I alpha 1; Dlx2, Distal-less homeobox2; qRT-PCR, real-time reverse transcription-polymerase chain reaction. 


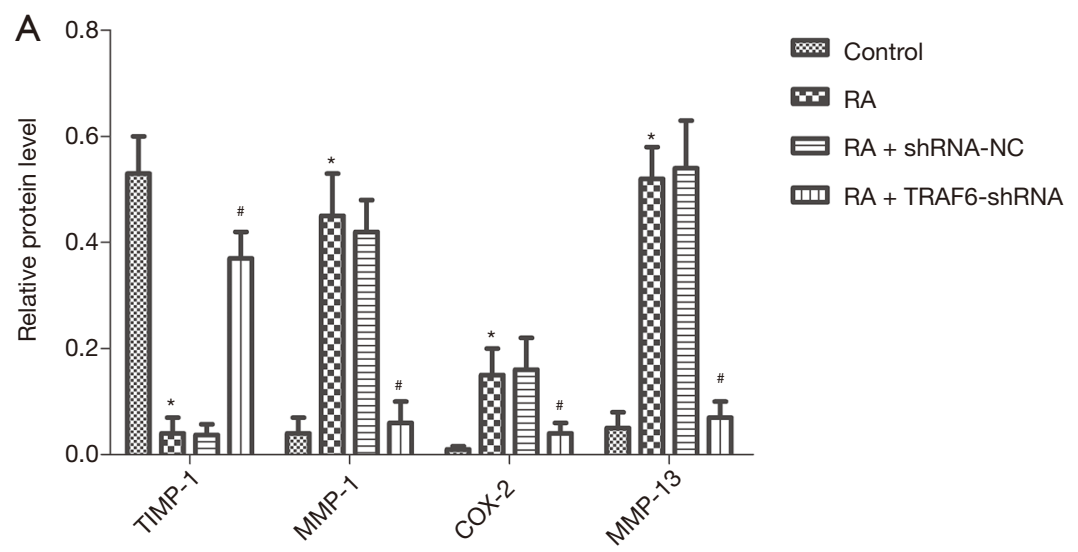

\section{B}
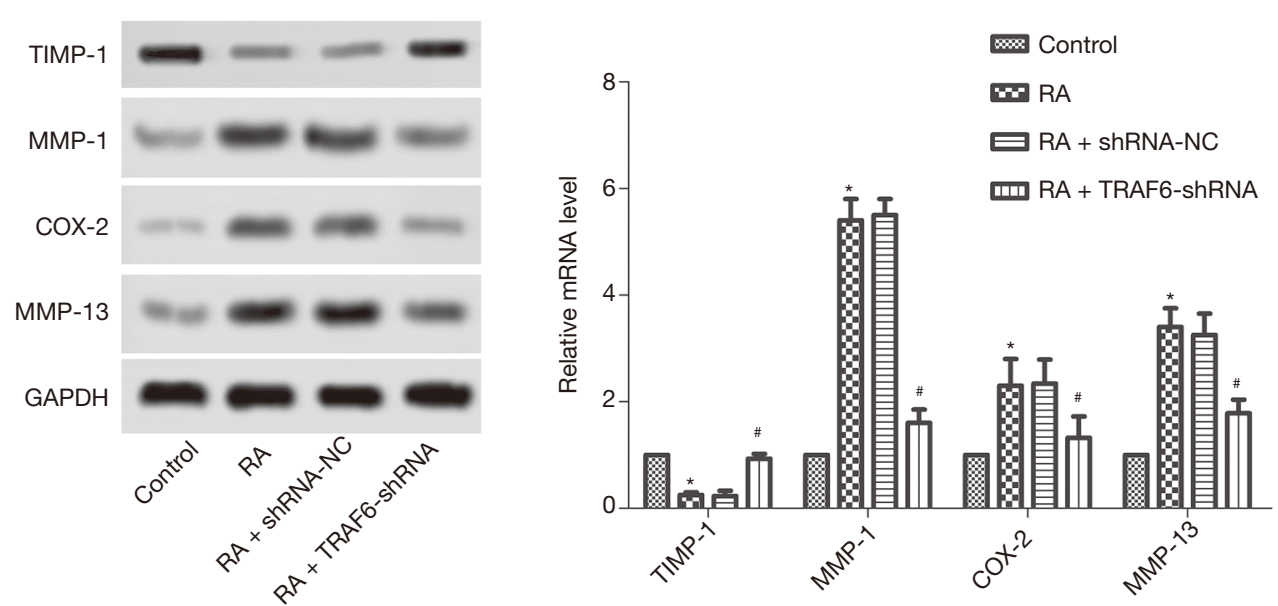

Figure 5 Effect of down-regulation of TRAF6 on MMPs. (A) The mRNA expressive levels of TIMP-1, MMP-1, COX2, and MMP-13 was performed by qRT-PCR; (B) the expressive protein levels of TIMP-1, MMP-1, COX2, and MMP-13 were performed by western blot. *, $\mathrm{P}<0.05$, vs. control group; ${ }^{*}, \mathrm{P}<0.05$, vs. the $\mathrm{RA}+$ shRNA-NC group. TRAF6, tumor necrosis factor receptor-associated factor; MMP, matrix metalloproteinase; qRT-PCR, real-time reverse transcription-polymerase chain reaction.

inhibited, the mRNA and protein expression levels of OSCAR and TRAP were reduced obviously in the RA + TRAF6-shRNA group compared with the RA + shRNANC group. Recently, a study has exhibited that OSCARcollagen signaling in monocytes plays a proinflammatory role and facilitates monocytes differentiation into osteoclasts and bone resorption and contributes to the pathogenesis of RA $(29,30)$. The TRAP isoform $5 \mathrm{~b}$ is a potential serum marker for osteoclastic activity (31). These results suggested that inhibition of TRAF6 suppressed osteoclast production in the development process of RA.

Bone homeostasis lies with the absorption of bone by osteoclasts and the formation of bone by osteoblasts. This imbalance in the tight coupling process can bring about diseases, including osteoporosis. Together, osteoblasts produce cytokines to regulate the differentiation and formation of osteoclasts. Also, osteoclast progenitor cells can reduce the sensitivity to osteoblast-induced apoptosis through the Fas-Ligand (FasL)/FAS pathway (32). Research has exhibited B cells restrain bone formation in RA by inhibiting osteoblast differentiation (33). The pathogenesis of RA is related to the inhibition of osteoblast differentiation. Restoring the function of osteoblasts plays a vital function in the treatment of RA. Interleukin (IL)-35 stimulates the differentiation of basal and TNF-activated osteoblasts via regulating the $\mathrm{Wnt} /$ catenin signaling pathway. Therefore, IL-35 has essential significance in the treatment of RA bone loss drugs and drug applications (34). 
OSX is an osteoblast-specific transcription factor, which is essential for osteoblast differentiation and bone formation. It is worth noting that OSX is the upstream regulator of Special AT-rich sequence-binding protein 2 (Satb2) during bone formation (35). CoL1A1driven transgenic markers are in the osteoblast lineage progression (36). DLX2 can induce Wnt1 transcription and regulate $\mathrm{Wnt} / \mathrm{catenin}$ signaling pathway to accelerate the osteogenic differentiation of human bone marrow mesenchymal stem cells (hBMSCs) (37). In our research, HE stains showed that osteoblast loss was observed in the RA group. However, after the levels of TRAF6 were inhibited, HE stains showed that osteoblast loss was recovered in the RA + TRAF6-shRNA group compared with the RA + shRNA-NC group. The result showed that the number of osteoblasts was suppressed significantly in the RA group. However, after the levels of TRAF6 were inhibited, the number of osteoblasts was elevated significantly in the RA + TRAF6-shRNA group compared with the RA + shRNA-NC group. The contents of cartilage and trabecular bone were markedly decreased in the RA group, but were increased after the levels of TRAF6 were inhibited in the RA + TRAF6-shRNA group compared with the RA + shRNA-NC group. The mRNA and protein expression levels of OSX, CoL1A1, and DLx2 were reduced in the RA group. However, after the levels of TRAF6 were inhibited, the mRNA and protein expression levels of OSX, CoL1A1, and DLx2 were enhanced obviously in the RA + TRAF6-shRNA group compared with the RA + shRNANC group. These results suggested that inhibition of TRAF6 promoted osteoblast production.

Cartilage degradation is put down to metalloproteinases (MPs) belonging to the MMP family; further, integrins and metalloproteinases with thrombospondin type 1 motifs produced by inflamed joint tissues (38). The expression of MMPs was detected in the joint ligaments, tendons, and cartilage tissues. They are known to contribute to the development, remodeling, and maintenance of healthy tissues through their ability to lyse various extracellular matrix substrates. Their role has been extended to cell growth, migration, differentiation, and apoptosis (39). Chondrocytes play a role in the development of RA through TMEM147-mediated NF- $\kappa \mathrm{B}$ activation and propose a treatment strategy for RA (40). Previous results have revealed that siTRAF6 can attenuate arthritis in mice with collagen-induced arthritis, as have evidenced by the reduction of serum anti-CII, MMP-1, MMP-3, and MMP-9 and the decreasing of histological damage. It is worth noting the blockade of TRAF6 inhibited the migration and invasion of human RA-FLSs with IL-1 $\beta$-stimulated (41). The correlation between TIMP-1 baseline levels and periarticular bone loss over one year suggests that TIMP-1 can be used as a biomarker of peri-articular bone loss in the early stage of RA (42).

Interestingly, RA synovial fibroblasts promote TREM-1 expression in monocytes via COX-2/prostaglandin E2 (PGE2) pathway (43). Kaempferol suppresses synovial fibroblast proliferation and the production of and MMPs, COX-2, and PGE2 in RA (44). In our research, the mRNA and protein expression levels of TIMP-1 were reduced obviously in the RA group, while increasing the mRNA and protein expression levels of MMP-1, COX2, and MMP-13. However, after the levels of TRAF6 were inhibited, The mRNA and protein expression levels of TIMP1were enhanced obviously in the RA + TRAF6-shRNA group compared with the RA + shRNA-NC group, while decreasing mRNA and protein expression levels of TIMP-1, MMP-1, COX-2 and MMP-13 $(\mathrm{P}<0.05)$. These results suggested that inhibition of TRAF6 improved MMPs production.

In conclusion, the down-regulation of TRAF6 restored the balance of osteoclasts and osteoblasts and improved the expression of MMP, which plays a vital role in the recovery of RA.

\section{Acknowledgments}

Funding: The Ningxia Natural Science Foundation Project supported this work (2020 AAC02035), National Natural Science Foundation of China Regional Science Foundation Project (81660281), Ningxia Hui Autonomous region Department of People's and Social Affairs overseas students innovation and entrepreneurship project (2017).

\section{Footnote}

Reporting Checklist: The authors have completed the ARRIVE reporting checklist. Available at http://dx.doi. org/10.21037/apm-20-1894

Data Sharing Statement: Available at http://dx.doi. org/10.21037/apm-20-1894

Conflicts of Interest: All authors have completed the ICMJE 
uniform disclosure form (available at http://dx.doi. org/10.21037/apm-20-1894). The authors have no conflicts of interest to declare.

Ethical Statement: The authors are accountable for all aspects of the work in ensuring that questions related to the accuracy or integrity of any part of the work are appropriately investigated and resolved. All animal care and handling were performed considering the National Institutes of Health (NIH) Guidelines for the Care and Use of Laboratory Animals and were approved by the People's Hospital of Ningxia Hui Autonomous Region. The research was reviewed and approved by the Ethics Committee for Experimental Animal Management and Animal Welfare of People's Hospital of Ningxia Hui Autonomous Region.

Open Access Statement: This is an Open Access article distributed in accordance with the Creative Commons Attribution-NonCommercial-NoDerivs 4.0 International License (CC BY-NC-ND 4.0), which permits the noncommercial replication and distribution of the article with the strict proviso that no changes or edits are made and the original work is properly cited (including links to both the formal publication through the relevant DOI and the license). See: https://creativecommons.org/licenses/by-nc-nd/4.0/.

\section{References}

1. Smolen JS, Aletaha D, McInnes IB. Rheumatoid arthritis. Lancet 2016;388:2023-38.

2. Wasserman A. Rheumatoid arthritis: common questions about diagnosis and management. Am Fam Physician 2018;97:455-62.

3. Paula FS, Alves JD. Non-tumor necrosis factor-based biologic therapies for rheumatoid arthritis: present, future, and insights into pathogenesis. Biologics 2014;8:1-12.

4. Trieb K. Treatment of the wrist in rheumatoid arthritis. J Hand Surg Am 2008;33:113-23.

5. Pisetsky DS. Advances in the Treatment of Rheumatoid Arthritis: Costs and Challenges. N C Med J 2017;78:337-40.

6. Pirmardvand Chegini S, Varshosaz J, Taymouri S. Recent approaches for targeted drug delivery in rheumatoid arthritis diagnosis and treatment. Artif Cells Nanomed Biotechnol 2018;46:502-14.

7. Pham CT. Nanotherapeutic approaches for the treatment of rheumatoid arthritis. Wiley Interdiscip Rev Nanomed Nanobiotechnol 2011;3:607-19.
8. Courties G, Presumey J, Duroux-Richard I, et al. RNA interference-based gene therapy for successful treatment of rheumatoid arthritis. Expert Opin Biol Ther 2009;9:535-8.

9. Kondo T, Kawai T, Akira S. Dissecting negative regulation of Toll-like receptor signaling. Trends Immunol 2012;33:449-58.

10. Wu H, Li XM, Wang JR, et al. NUR77 exerts a protective effect against inflammatory bowel disease by negatively regulating the TRAF6/TLR-IL-1R signalling axis. J Pathol 2016;238:457-69.

11. Huang D, Bai Z, Shen J, et al. Identification of tumor necrosis factor receptor-associated factor 6 in the pearl mussel Hyriopsis cumingii and its involvement in innate immunity and pearl sac formation. Fish Shellfish Immunol 2018;80:335-47.

12. Fang J, Bolanos LC, Choi K, et al. Ubiquitination of hnRNPA1 by TRAF6 links chronic innate immune signaling with myelodysplasia. Nat Immunol 2017;18:236-45.

13. Chen H, Li M, Sanchez E, et al. Combined TRAF6 Targeting and Proteasome Blockade Has Anti-myeloma and Anti-Bone Resorptive Effects. Mol Cancer Res 2017;15:598-609.

14. Sun H, Li XB, Meng Y, et al. TRAF6 upregulates expression of HIF-1 $\alpha$ and promotes tumor angiogenesis. Cancer Res 2013;73:4950-9.

15. Wu H, Lu XX, Wang JR, et al. TRAF6 inhibits colorectal cancer metastasis through regulating selective autophagic CTNNB1/ $\beta$-catenin degradation and is targeted for GSK3B/GSK3 $\beta$-mediated phosphorylation and degradation. Autophagy 2019;15:1506-22.

16. Rezaeian AH, Li CF, Wu CY, et al. A hypoxia-responsive TRAF6-ATM-H2AX signalling axis promotes HIF1 $\alpha$ activation, tumorigenesis and metastasis. Nat Cell Biol 2017;19:38-51.

17. Zhu L, Ouyang X, Zheng D, et al. Correlation between synovial TRAF6 expression and serum bone metabolism markers in rheumatoid arthritis. Zhonghua Yi Xue Za Zhi 2014;94:1643-6.

18. Zhang HC, Liu MX, Wang EP, et al. Effect of sinomenine on the expression of rheumatoid arthritis fibroblastlike synoviocytes MyD88 and TRAF6. Genet Mol Res 2015;14:18928-35.

19. Hu B, Sun X, Yang Y, et al. Tomatidine suppresses osteoclastogenesis and mitigates estrogen deficiencyinduced bone mass loss by modulating TRAF6-mediated signaling. FASEB J 2019;33:2574-86.

20. Wang X, Yan X, Wang F, et al. Role of methotrexate 
chronotherapy in collagen-induced rheumatoid arthritis in rats. Z Rheumatol 2018;77:249-55.

21. Baharav E, Mor F, Halpern M, et al. Lactobacillus GG bacteria ameliorate arthritis in Lewis rats. J Nutr 2004;134:1964-9.

22. Zhu LJ, Yang TC, Wu Q, et al. Tumor necrosis factor receptor-associated factor (TRAF) 6 inhibition mitigates the pro-inflammatory roles and proliferation of rheumatoid arthritis fibroblast-like synoviocytes. Cytokine 2017;93:26-33.

23. Shiozawa S, Tsumiyama K, Yoshida K, et al. Pathogenesis of joint destruction in rheumatoid arthritis. Arch Immunol Ther Exp (Warsz) 2011;59:89-95.

24. Boyce BF. Advances in the regulation of osteoclasts and osteoclast functions. J Dent Res 2013;92:860-7.

25. Tanaka S. Emerging anti-osteoclast therapy for rheumatoid arthritis. J Orthop Sci 2018;23:717-21.

26. Lamothe B, Webster WK, Gopinathan A, et al. TRAF6 ubiquitin ligase is essential for RANKL signaling and osteoclast differentiation. Biochem Biophys Res Commun 2007;359:1044-9.

27. Hassine HB, Zemni R, Nacef IB, et al. A TRAF6 genetic variant is associated with low bone mineral density in rheumatoid arthritis. Clin Rheumatol 2019;38:1067-74.

28. Zhu LJ, Dai L, Zheng DH, et al. Upregulation of tumor necrosis factor receptor-associated factor 6 correlated with synovitis severity in rheumatoid arthritis. Arthritis Res Ther 2012;14:R133.

29. Schultz HS, Guo L, Keller P, et al. OSCAR-collagen signaling in monocytes plays a proinflammatory role and may contribute to the pathogenesis of rheumatoid arthritis. Eur J Immunol 2016;46:952-63.

30. Herman S, Müller RB, Krönke G, et al. Induction of osteoclast-associated receptor, a key osteoclast costimulation molecule, in rheumatoid arthritis. Arthritis Rheum 2008;58:3041-50.

31. Janckila AJ, Takahashi K, Sun SZ, et al. NaphtholASBI phosphate as a preferred substrate for tartrateresistant acid phosphatase isoform 5b. J Bone Miner Res 2001;16:788-93.

32. Chen X, Wang Z, Duan N, et al. Osteoblast-osteoclast interactions. Connect Tissue Res 2018;59:99-107.

33. Sun W, Meednu N, Rosenberg A, et al. B cells inhibit bone formation in rheumatoid arthritis by suppressing osteoblast differentiation. Nat Commun 2018;9:5127.

34. Li Y, Yuan L, Jiang S, et al. Interleukin-35 stimulates tumor necrosis factor- $\alpha$ activated osteoblasts differentiation through $W \mathrm{nt} / \beta$-catenin signaling pathway in rheumatoid arthritis. Int Immunopharmacol 2019;75:105810.

35. Tang W, Li Y, Osimiri L, et al. Osteoblast-specific transcription factor Osterix (OSX) is an upstream regulator of Satb2 during bone formation. J Biol Chem 2011;286:32995-3002.

36. Dacic S, Kalajzic I, Visnjic D, et al. Col1a1-driven transgenic markers of osteoblast lineage progression. J Bone Miner Res 2001;16:1228-36.

37. Zeng X, Wang Y, Dong Q, et al. DLX2 activates Wnt1 transcription and mediates $W n t / \beta$-catenin signal to promote osteogenic differentiation of hBMSCs. Gene 2020;744:144564.

38. Itoh Y. Metalloproteinases in rheumatoid arthritis: potential therapeutic targets to improve current therapies. Prog Mol Biol Transl Sci 2017;148:327-38.

39. Bramono DS, Richmond JC, Weitzel PP, et al. Matrix metalloproteinases and their clinical applications in orthopaedics. Clin Orthop Relat Res 2004:272-85.

40. Ota M, Tanaka Y, Nakagawa I, et al. Role of Chondrocytes in the Development of Rheumatoid Arthritis Via Transmembrane Protein 147-Mediated NF- $\kappa B$ Activation. Arthritis Rheumatol 2020;72:931-42.

41. Wang H, Chen W, Wang L, et al. Tumor necrosis factor receptor-associated factor 6 promotes migration of rheumatoid arthritis fibroblast-like synoviocytes. Mol Med Rep 2015;11:2761-6.

42. Murphy E, Roux-Lombard P, Rooney T, et al. Serum levels of tissue inhibitor of metalloproteinase- 1 and periarticular bone loss in early rheumatoid arthritis. Clin Rheumatol 2009;28:285-91.

43. Peng A, Lu X, Huang J, et al. Rheumatoid arthritis synovial fibroblasts promote TREM-1 expression in monocytes via COX-2/PGE(2) pathway. Arthritis Res Ther 2019;21:169.

44. Yoon HY, Lee EG, Lee H, et al. Kaempferol inhibits IL$1 \beta$-induced proliferation of rheumatoid arthritis synovial fibroblasts and the production of COX-2, PGE2 and MMPs. Int J Mol Med 2013;32:971-7.

(English Language Editor: J. Chapnick)

Cite this article as: Guo J, Cao X, Ma X, Hao C, Wu L, Zhang M, Yang Y, Zhao J, Chen K, Yin Z. Tumor necrosis factor receptor-associated factor 6 (TRAF6) inhibition modulates bone loss and matrix metalloproteinase expression levels in collagen-induced rheumatoid arthritis rat. Ann Palliat Med 2020;9(6):4017-4028. doi: 10.21037/apm-20-1894 